\title{
Fronts from integrodifference equations and persistence effects on the Neolithic transition
}

\author{
Joaquim Fort, Joaquim Pérez-Losada, and Neus Isern \\ Departament de Física, Universitat de Girona, 17071 Girona, Catalonia, Spain
}

(Received 5 January 2007; revised manuscript received 31 July 2007; published 14 September 2007)

\begin{abstract}
We extend a previous model of the Neolithic transition in Europe [J. Fort and V. Méndez, Phys. Rev. Lett. 82, 867 (1999)] by taking two effects into account: (i) we do not use the diffusion approximation (which corresponds to second-order Taylor expansions), and (ii) we take proper care of the fact that parents do not migrate away from their children (we refer to this as a time-order effect, in the sense that it implies that children grow up with their parents, before they become adults and can survive and migrate). We also derive a time-ordered, second-order equation, which we call the sequential reaction-diffusion equation, and use it to show that effect (ii) is the most important one, and that both of them should in general be taken into account to derive accurate results. As an example, we consider the Neolithic transition: the model predictions agree with the observed front speed, and the corrections relative to previous models are important (up to $70 \%$ ).
\end{abstract}

DOI: $10.1103 /$ PhysRevE.76.031913

PACS number(s): 87.23.Cc, 89.20.-a, 89.75.Fb

\section{INTRODUCTION}

Reaction-diffusion fronts are important in many areas of physics, including biological and cross-disciplinary systems $[1,2]$. Some examples are Neolithic transition fronts [3-9], virus infection fronts [10,11], biological invasions [12], combustion flames [13-16], tumor growth [17-19], etc.

As in previous work $[1,3,10,11]$, we shall here consider a two-dimensional (2D) space. This will be necessary in order to apply our results to a specific example, namely, the Neolithic transition.

Up to now, most of the front solutions analyzed come from evolution equations that rely on the assumption that second-order Taylor expansions are valid approximations. This may be seen as follows. Let $p(x, y, t)$ stand for the population (or particle) number per unit area at position $(x, y)$ and time $t$. The dispersal kernel $\phi\left(\Delta_{x}, \Delta_{y}\right)$ is the probability per unit area that an individual (or particle) who was at $\left(x+\Delta_{x}, y+\Delta_{y}, t\right)$ jumps to $(x, y, t+T)$. Here $T$ is the time interval between two subsequent jumps (in biophysical applications, usually $T=1$ generation $[3,10])$. Let $R[p(x, y, t)]$ stand for the new individuals (or particles) due to the reproduction process (or chemical reactions), produced during the time interval $T$ per unit area centered at $(x, y)$. From these definitions, the evolution equation is typically written as follows [3]:

$$
\begin{aligned}
& p(x, y, t+T)-p(x, y, t) \\
& =\int_{-\infty}^{+\infty} \int_{-\infty}^{+\infty} p\left(x+\Delta_{x}, y+\Delta_{y}, t\right) \phi\left(\Delta_{x}, \Delta_{y}\right) d \Delta_{x} d \Delta_{y} \\
& \quad-p(x, y, t)+R[p(x, y, t)]
\end{aligned}
$$

where the first and second terms on the right-hand side correspond to population dispersal, and the last one to reproduction. An age-structured derivation of Eq. (1) is included in Appendix A. There, it is also shown that $R[p(x, y, t)]$ in Eq. (1) includes the effect of deaths besides reproduction. Thus, $R[p(x, y, t)]$ is a net reproductive term.
Usually second-order Taylor expansions in space (diffusion) and time are performed. Then, assuming an isotropic kernel, i.e., that $\phi\left(\Delta_{x}, \Delta_{y}\right)$ depends only on

$$
\Delta=\sqrt{\Delta_{x}^{2}+\Delta_{y}^{2}}
$$

the integrodifference equation (1) becomes a partial differential equation, namely [3],

$$
\frac{\partial p}{\partial t}+\frac{T}{2} \frac{\partial^{2} p}{\partial t^{2}}=D\left(\frac{\partial^{2} p}{\partial x^{2}}+\frac{\partial^{2} p}{\partial y^{2}}\right)+F+\frac{T}{2} \frac{\partial F}{\partial t} .
$$

Equation (3) is called the hyperbolic reaction-diffusion (HRD) equation [1] (it becomes the telegraph equation if $F$ $=0) . F$ is the time derivative of $p(x, y, t)$ due to reproduction, i.e., $[3]$

$$
R[p(x, y, t)]=T F+\frac{T^{2}}{2 !} \frac{\partial F}{\partial t}+\frac{T^{3}}{3 !} \frac{\partial^{2} F}{\partial t^{2}}+\cdots,
$$

and we have also introduced the diffusion coefficient

$$
D=\frac{1}{4 T} \int_{-\infty}^{+\infty} \int_{-\infty}^{+\infty} \phi(\Delta) \Delta^{2} d \Delta_{x} d \Delta_{y} \equiv \frac{\left\langle\Delta^{2}\right\rangle}{4 T} .
$$

Usually, front speeds derived analytically from a partial differential equation such as (3) are compared to the numerical simulations of the same differential equation. Agreement between both approaches is considered a useful check of the results $[3,10]$. It is true that almost all papers on front propagation follow Eq. (3) (or its parabolic limit, i.e., $T \rightarrow 0$, which yields Fisher's equation and corresponds to Fickian diffusion). However, such a procedure has two main limitations.

(i) On the one hand, there is no reason a priori to expect that Eq. (3) is a valid approximation to the original Eq. (1). Therefore, in the present paper we will go beyond the second-order (HRD) approximation.

(ii) We will also take into account an additional point that will make our results more realistic than previous ones, as follows. According to the last term in Eq. (1), the population at $(x, y, t)$ reproduces causing a population number increase at the same space point $(x, y)$ one generation later $(t+T)$. But, in fact, that parent population will have migrated out of 
$(x, y)$ at time $t+T$ because of the second term on the righthand side of Eq. (1). In other words, parents migrate away from their children according to Eq. (1). This is appropriate for some biological species (e.g., fish) but not for humans. Indeed, according to anthropological observations, children typically live with their parents until they become adults and can survive (and migrate). Therefore, for human populations it is more realistic to replace Eq. (1) by

$$
\begin{aligned}
p(x, y, t+T)= & \int_{-\infty}^{+\infty} \int_{-\infty}^{+\infty} p\left(x+\Delta_{x}, y+\Delta_{y}, t\right) \phi\left(\Delta_{x}, \Delta_{y}\right) d \Delta_{x} d \Delta_{y} \\
& +R\left(\int _ { - \infty } ^ { + \infty } \int _ { - \infty } ^ { + \infty } p \left(x+\Delta_{x}, y\right.\right. \\
& \left.\left.+\Delta_{y}, t\right) \phi\left(\Delta_{x}, \Delta_{y}\right) d \Delta_{x} d \Delta_{y}\right)
\end{aligned}
$$

so that after the individuals of the population move [once per generation, from $\left(x+\Delta_{x}, y+\Delta_{y}\right)$ into $(x, y)$, first line in Eq. (6)], they reproduce at the arrival location $(x, y)$ [second line in Eq. (6)]. An age-structured derivation of Eq. (6) is included in Appendix B.

Equation (6) assumes that parents first migrate and then reproduce. An alternative to Eq. (6) is to assume that parents first reproduce and then migrate carrying their children with them [not leaving them alone as it happened with Eq. (1)]. In this case we have, instead of Eq. (6),

$$
\begin{aligned}
p(x, y, t+T)= & \int_{-\infty}^{+\infty} \int_{-\infty}^{+\infty} p\left(x+\Delta_{x}, y+\Delta_{y}, t\right) \phi\left(\Delta_{x}, \Delta_{y}\right) d \Delta_{x} d \Delta_{y} \\
& +\int_{-\infty}^{+\infty} \int_{-\infty}^{+\infty} R\left[p\left(x+\Delta_{x}, y+\Delta_{y}, t\right)\right] \\
& \times \phi\left(\Delta_{x}, \Delta_{y}\right) d \Delta_{x} d \Delta_{y} .
\end{aligned}
$$

However, we shall see in Sec. II A that for our purposes, both Eqs. (6) and (7) give the same front speed (this can be also shown for another case, namely, simultaneous migration and reproduction, but it has a negligible effect since this happens very rarely for humans-because migrations have a duration of hours or days, whereas the time scale of reproduction is 1 generation $\simeq 32$ years [5]).

The important difference is that for Eq. (1) parents migrate away from their children, whereas for Eqs. (6) and (7) they do not. So the latter are more realistic for humans.

Some recent work on front speeds goes beyond the second-order approximation [22-24] [point (i) above]. But those papers use evolution equations of the form (1) instead of (6). Thus, the fact that children grow up with their parents [point (ii) above] was not properly taken into account in those references.

Concerning the function $R[p(x, y, t)]$, which corresponds to the effect of population reproduction, we next summarize some of the many choices that have been considered in the literature.

(i) The so-called exponential growth function for $F$ appearing in Eq. (4), namely [3] (see also Appendixes A and B of the present paper),

$$
F=r_{L} p(x, y, t),
$$

where $r_{L}$ is called the intrinsic rate of growth. This case leads to population densities increasing in time without any bound, which is not realistic biologically.

(ii) The logistic growth function for $F$ appearing in Eq. (4), namely [3],

$$
F=a p(x, y, t)\left(1-\frac{p(x, y, t)}{p_{\max }}\right),
$$

where $a$ is called the initial growth rate. This case predicts a bounded population growth, but it is extremely troublesome for numerical implementation, because it involves an infinite number of terms [see Eq. (4)]. Since we want to check our results numerically, we will not use this choice for $F$ in the present paper.

(iii) The logistic form (8) cannot be applied for $R[p(x, y, t)]$ instead of $F$ neither, because it is known from nonspatial models [40] that it would then yield negative values for the population number density $p(x, y, t)$, which is unphysical.

(iv) We think that a very reasonable choice is to assume reproduction proportional to the population density, but bounded by a maximum value, $p_{\max }$, due to the environmental limitations, i.e.,

$$
R[p(x, y, t)]= \begin{cases}\left(R_{0}-1\right) p(x, y, t) & \text { if } p<p_{\max }, \\ 0 & \text { if } p \geq p_{\max }\end{cases}
$$

where $R_{0}$ is called the net reproductive rate (or fecundity) per generation, and $p_{\max }$ is the saturation density [25].

The difference between an exponential $F$, a logistic $F(8)$ and Eq. (9) is not significant concerning invasion front speeds. Concerning reproduction, they all imply an exponential increase at low values of $p$ [26], and both (8) and (9) imply that population growth ceases as $p \rightarrow p_{\max }^{-}$. The manner in which the latter happens does not change the speed of fronts [27]. It has not been determined from observations [28]. Also, Eq. (9) is simpler for numerical implementation (Sec. III). For these reasons and the difficulties mentioned for the choices (i)-(iii), we use here Eq. (9).

The rest of the paper is organized as follows. In Sec. II we derive the speed of fronts for Eq. (6) using a continuousspace random-walk (CSRW) model using parameter values appropriate for the phenomenon we are interested in, namely, the Neolithic transition in Europe [3]. In Sec. III we shall develop computer simulations and discrete-space random walks (DSRWs) to check the results from the CSRWs. In Sec. IV we discuss the implications of the results to a specific example, namely the Neolithic transition in Europe. We also compare the predictions of Eqs. (6) and (3). In Sec. V we derive a reaction-diffusion equation. We call it the sequential reaction-diffusion (SRD) equation. It is analogous to the HRD equation (3) but improves it, by taking care of the time order of events (in the sense that children grow up with their parents until they become adults and can survive and migrate). Section VI is devoted to concluding remarks. 


\section{CONTINUOUS-SPACE RANDOM-WALK MODEL}

\section{A. The speed of fronts}

For low values of the population density, i.e., for first line in Eq. (9), Eq. (6) becomes

$$
\begin{aligned}
p(x, y, t+T)= & R_{0} \int_{-\infty}^{+\infty} \int_{-\infty}^{+\infty} p\left(x+\Delta_{x}, y\right. \\
& \left.+\Delta_{y}, t\right) \phi\left(\Delta_{x}, \Delta_{y}\right) d \Delta_{x} d \Delta_{y}
\end{aligned}
$$

(for an age-structured derivation of this equation, see Appen$\operatorname{dix} \mathrm{C})$.

Note that using Eq. (7) instead of (6), we again obtain Eq. (10) provided that the net reproductive rate $R_{0}$ is independent of the jump length vector $\left(\Delta_{x}, \Delta_{y}\right)$. We think this is a reasonable assumption, and are not aware of any anthropological data suggesting otherwise. Therefore, our model is valid both for migration preceding reproduction, and for reproduction preceding migration [as well as for reproduction simultaneous with migration - this is easily seen but it is very rare in humans, as explained below Eq. (7)].

Equations similar to (6) have been previously considered and applied to predict front speeds, but only in onedimensional space $[20,21]$. Here we will deal with the twodimensional case, as necessary for application to the Neolithic transition. Also, for the first time we shall apply the integrodifference Eq. (6) to the Neolithic transition, and compare the results to those from the differential (HRD) equation (3), which was used in previous work on the Neolithic transition [3].

In order to derive analytical results, we first recall that the speed (for azimuthally symmetric front solutions) can be found most easily by assuming that for $t \rightarrow \infty$ the front curvature is negligible at scales much larger than that of individual dispersal events. In other words, we assume that we can choose the $x$ axis parallel to the local velocity of the front, an approach that has been successful in previous work $[1,22]$. Let $c \equiv\left|c_{x}\right|$ stand for the front speed $\left(c_{y}=0\right.$ in the local frame just introduced). We look for constant-shape solutions with the form $p=p_{0} \exp [-\lambda(x-c t)]$ as $x-c t \rightarrow \infty$. Then, assuming an isotropic kernel $\phi(\Delta)$, we obtain from Eq. (10),

$$
\exp (c T \lambda)=R_{0} \int_{0}^{\infty} d \Delta \Delta \phi(\Delta) \int_{0}^{2 \pi} d \theta \exp (-\lambda \Delta \cos \theta)
$$

where we have introduced

$$
\theta \equiv \tan ^{-1} \frac{\Delta_{y}}{\Delta_{x}}
$$

In order to perform the integrals, we need an expression for the kernel $\phi(\Delta)$. There are many possible choices of the kernel. Here we are interested in a single, the simplest possible kernel such that we can perform numerical simulations and derive analytical formulas. For this reason, we simply assume that an individual will either remain at rest (with probability $p_{e}$, which is called the persistence in demography) or will move a distance $r$ (with probability $1-p_{e}$ ),

$$
\begin{aligned}
\phi(\Delta) & =p_{e} \delta^{(2)}(\Delta)+\left(1-p_{e}\right) \delta^{(2)}(\Delta-r) \\
& =p_{e} \frac{\delta^{(1)}(\Delta)}{2 \pi \Delta}+\left(1-p_{e}\right) \frac{\delta^{(1)}(\Delta-r)}{2 \pi \Delta}
\end{aligned}
$$

where $\delta^{(2)}(\Delta-r)$ is the two-dimensional Dirac $\delta$ centered at $\Delta=r$, whereas $\delta^{(1)}(\Delta-r)$ is the one-dimensional Dirac $\delta$ centered at $\Delta=r[29]$.

Therefore, in this simple model we assume that all moving individuals travel the same distance $r$. This will make it possible to find relatively simple analytical results and to run relatively fast random-walk simulations.

We perform the integrals and, as usual, assume that the minimum speed is the one of the front $[1,2]$ (we will check this assumption by means of numerical simulations in Sec. III). In this way an explicit expression for the speed is obtained,

$$
c=\min _{\lambda>0} \frac{\ln \left\{R_{0}\left[p_{e}+\left(1-p_{e}\right) I_{0}(\lambda r)\right]\right\}}{T \lambda},
$$

where

$$
I_{0}(\lambda r) \equiv \frac{1}{2 \pi} \int_{0}^{2 \pi} d \theta \exp (\lambda r \cos \theta)
$$

is the modified Bessel function of the first kind and order zero.

\section{B. Parameter estimates and predicted speed}

In Fig. 1, we show the speed predicted by the CSRW (full line), Eq. (14), for values of $R_{0}, p_{e}, T$, and $r$ typical of human Neolithic populations. The parameter values have been estimated as follows. The generation time has been carefully estimated, in a very recent paper [5], as $T=1$ generation $=32 \mathrm{yr}$. To estimate $R_{0}$, we recall that Birdsell was able to collect population number growth data on two human populations that settled in previously unpopulated areas [30]. What is impressive of those data is that, when plotted against the elapsed time in generations, both data sets yield almost exactly the same curve of population number, relative to the initial value [i.e., $p(x, y, t) / p(x, y, t=0)]$ versus time. From Eq. (10) in the absence of dispersion effects, we can estimate the value of $R_{0}=p(x, y, t+T) / p(x, y, t)$ for several values of $t=T, 2 T, 3 T, \ldots$. This yields an average of $R_{0}=2.2$. The lowest value is $R_{0}=1.9$, and the highest one is $R_{0}=2.6$. Thus, in Fig. 1 we compute front speeds for values of $R_{0}$ in the range 1.6-3.0. On the other hand, we have estimated the population persistence $p_{e}$ (i.e., the fraction of the population that does not move appreciably) directly from the mobility data in Ref. [31], p. 139, for three different populations of preindustrial agriculturalists. The corresponding values of $p_{e}$ are 0.54, 0.40, and 0.19. In Fig. 1, we use the mean value $p_{e}$ $=0.38$ (the dependence of the front speed of $p_{e}$ shall be analyzed later, in Fig. 3). Finally, the value of $r$ is estimated directly from that of the persistence and the mean-squared displacement [32].

In Fig. 1, the front speed predicted by the CSRW (full line), Eq. (14), is seen to increase with increasing values of the population fecundity, as it was to be expected intuitively. 


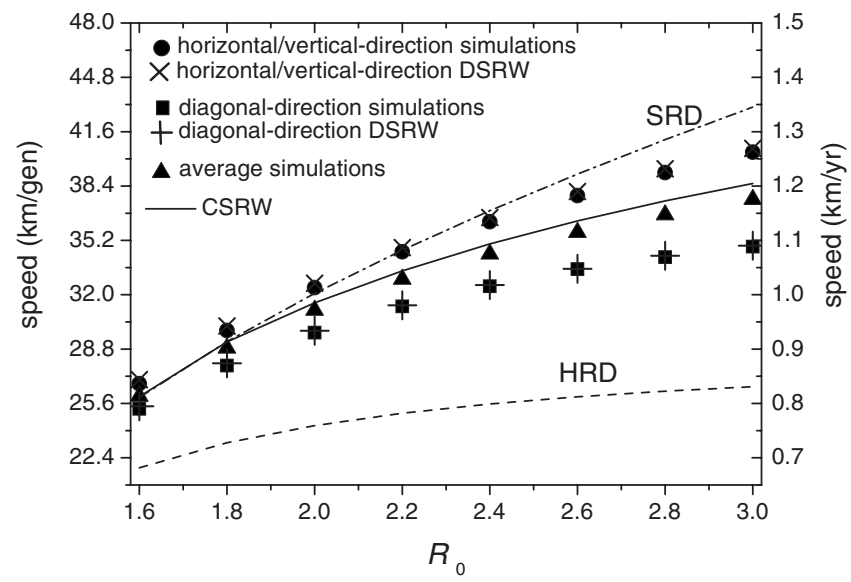

FIG. 1. Front speed in 2D versus population net reproductive rate. The numerical simulations in Sec. III lead to different speeds in the horizontal or vertical directions (circles) than in the diagonal directions (squares), but their average (triangles) agrees with the CSRW derived in Sec. II (full curve). Figure 2 explains intuitively why the speeds obtained from the simulations depend on the direction considered. The DSRW results $(X$ and + crosses $)$ agree perfectly with the simulations in the corresponding direction. This provides an accurate check of the simulation results. The prediction from the HRD approximation, derived in Ref. [3] (dashed curve), and that of the SRD one, derived in Sec. V (dashed-dotted curve), are shown for comparison.

Before analyzing the implications for the Neolithic transition and comparing to previous work, we first perform numerical simulations in order to check the validity of the result (14), which in turn relies on several assumptions (as mentioned above).

\section{DISCRETE-SPACE APPROACHES}

\section{A. Reactive random-walk simulations}

Obviously we must follow a very different approach to that in Refs. [1,33-36]. In all of those references, differential equations [e.g., (3)] were numerically integrated, by replacing derivatives by finite differences (e.g., $\frac{\partial p}{\partial t} \rightarrow \frac{\Delta p}{\Delta t}$ ). But in the present paper, we are no longer dealing with any approximate differential equation. Instead, Eq. (10) is an exact integrodifference equation. In other words, no derivatives appear at all because Taylor expansion approximations have not been performed. Therefore, our approach here cannot be based on finite differences. Instead, we must follow the space-time evolution of individuals, or of the population density $p(x, y, t)$, without making any such approximation. We shall, in this sense, refer to molecular-dynamics simulations, to distinguish the simulations in the present work from the finite-difference simulations in previous literature [1,33-36].

We consider a $2 \mathrm{D}$ grid with $10^{3} \times 10^{3}$ nodes. Initially $p(x, y, 0)=1$ at the central node, and 0 elsewhere. At each time step (corresponding to $T=1$ generation), we compute the new population number density $p(x, y, t+T)$ at all nodes of the 2D grid as follows.

(i) First we compute the new local population density due to reproduction at every node as $R_{0} p(x, y, t)$ if $p<p_{\max }$ [Eq.

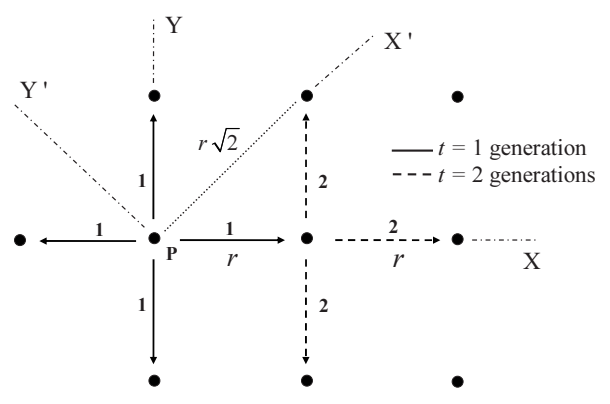

FIG. 2. Jump of individuals (or particles) for the square lattice in the simulations and the kernel considered, from an initial point $P$. Continuous arrows correspond to the first generation, whereas dotted arrows correspond to the second generation. After two generations, it is seen that the distance in the diagonal direction $(r \sqrt{2})$ is lower than that in the horizontal direction $(2 r)$. This is the intuitive reason why the random-walk simulation speeds in the horizontal or vertical direction (dots in Fig. 1) are observed to be higher than those in the diagonal direction (squares in Fig. 1). Analytically, we prove this statement by means of the DSRW (Sec. IV), which closely agrees with the simulations (see Fig. 1).

(10)] and $p_{\max }$ otherwise [second line in Eq. (9)]. This may be referred to as the reactive step of the simulations.

(ii) Then we redistribute this value among all grid nodes using the kernel (13). Thus, in this dispersive step, we consider that a fraction $p_{e}$ of the population stays at the original node, and the remaining fraction is distributed equally among the nearest neighbors, i.e., a fraction $\left(1-p_{e}\right) / 4$ jumps a distance $\pm r$ along each horizontal or vertical direction.

In the horizontal and/or vertical directions, the speed obtained from the simulations (circles in Fig. 1) is up to 5\% higher than that predicted by the CSRW (full line). But if we measure the speed along a diagonal $\left(45^{\circ}\right)$ direction (squares in Fig. 1), it is lower than the CSRW. The average of both results (triangles) agrees within about $2 \%$ with the analytical result from the CSRW (full line) [37-39]. Why does the speed from the random-walk simulations depend on the direction? Because unlike the CSRW, they are not isotropic (motion is only allowed in the horizontal and vertical directions). As shown in Fig. 2, after the first-generation jumps (full arrows), individuals reproduce. After two generations (dashed arrows), the dispersal distance along the diagonal direction $(r \sqrt{2})$ is lower than that in the horizontal direction $(2 r)$. We think this is the intuitive reason why the simulation speeds in the diagonal directions (squares in Fig. 1) are lower than in the horizontal and/or vertical directions (circles in Fig. 1). Indeed, we proof this statement analytically in the next section.

\section{B. Discrete-space random walks}

Here we present a discrete-space analog to the continuous-space analytical approach in Sec. II. It will be useful in order to test direction-dependent speeds observed in the simulations above.

\section{Horizontal/vertical direction $\left(0^{\circ}\right)$}

We first choose the $X$ and $Y$ axes shown in Fig. 2. Then, for the kernel (13), particles can jump into point $(x, y)$ from 
points $(x \pm r, y)$ and $(x, y \pm r)$. Therefore, Eq. (10) is replaced by

$$
\begin{aligned}
p(x, y, t+T)= & R_{0}\left[p_{e} p(x, y, t)+\left(1-p_{e}\right)\left(\frac{1}{4} p(x-r, y, t)+\frac{1}{4} p(x\right.\right. \\
& \left.\left.+r, y, t)+\frac{1}{4} p(x, y-r, t)+\frac{1}{4} p(x, y+r, t)\right)\right] .
\end{aligned}
$$

As in Sec. II, we look for solutions with the form $p$ $=p_{0} \exp [-\lambda(x-c t)]$ and assume that the minimum speed is the one of the front $[1,2]$. In this way we obtain the speed

$$
c=\min _{\lambda>0} \frac{\ln \left[R_{0}\left(\frac{p_{e}+1}{2}+\frac{1-p_{e}}{2} \cosh (\lambda r)\right)\right]}{\lambda T} .
$$

This equation has no analytical solution. However, for given values of $R_{0}, p_{e}, r$, and $T$ it is easy to find its minimum numerically. In this way we obtain the crosses in Fig. 1. They agree almost perfectly with the horizontal- and/or verticaldirection simulations, performed in the preceding section (circles in Fig. 1). This confirms the validity of the simulations in Sec. II.

\section{Diagonal direction $\left(45^{\circ}\right)$}

Now we choose $X^{\prime}$ and $Y^{\prime}$ axes shown in Fig. 2. Then, for the same kernel, it is easily seen that particles can jump into point $\left(x^{\prime}, y^{\prime}\right)$ from points $\left(x^{\prime} \pm \frac{r}{\sqrt{2}}, y^{\prime} \pm \frac{r}{\sqrt{2}}\right)$. Therefore, instead of Eq. (16) we have

$$
\begin{aligned}
p\left(x^{\prime}, y^{\prime}, t+T\right) & \\
= & R_{0}\left\{p_{e} p\left(x^{\prime}, y^{\prime}, t\right)+\left(1-p_{e}\right)\left[\frac{1}{4} p\left(x^{\prime}+\frac{r}{\sqrt{2}}, y^{\prime}+\frac{r}{\sqrt{2}}, t\right)\right.\right. \\
& +\frac{1}{4} p\left(x^{\prime}+\frac{r}{\sqrt{2}}, y^{\prime}-\frac{r}{\sqrt{2}}, t\right)+\frac{1}{4} p\left(x^{\prime}-\frac{r}{\sqrt{2}}, y^{\prime}+\frac{r}{\sqrt{2}}, t\right) \\
& \left.\left.+\frac{1}{4} p\left(x^{\prime}-\frac{r}{\sqrt{2}}, y^{\prime}--\frac{r}{\sqrt{2}}, t\right)\right]\right\},
\end{aligned}
$$

which leads us, in the same way, to the speed

$$
c=\min _{\lambda>0} \frac{\ln \left\{R_{0}\left[p_{e}+\left(1-p_{e}\right) \cosh \left(\lambda \frac{r}{\sqrt{2}}\right)\right]\right\}}{\lambda T},
$$

instead of (17). This speed is shown as crosses (+) in Fig. 1. It agrees perfectly with the diagonal-direction simulations (squares in Fig. 1).

The agreement between the DSRW model and the simulations (Fig. 1) confirms the validity of the lattice simulations in Sec. III A, as well as the direction dependence of the front speed on discrete spaces.

\section{APPLICATION TO THE NEOLITHIC TRANSITION IN EUROPE}

For the Neolithic transition, in Ref. [3], the second-order approximation (3) was applied. In contrast, the models in the present paper do not use any second-order approximation.

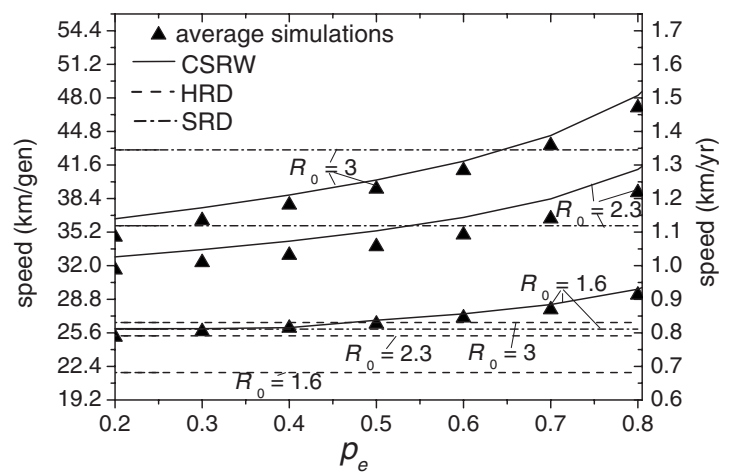

FIG. 3. The effect of dispersion persistence on the speed of the Neolithic transition. As in Fig. 1, the SRD equation is seen to be a better approximation than the HRD equation (which was used in Ref. [3]). The reason is that the SRD equation, derived in the present paper, takes proper care of the time order of events, whereas the HRD equation does not.

The front speed predicted from the second-order (HRD) approximation (3) and logistic population number growth (8) is [3]

$$
c_{\mathrm{HRD}}=\frac{2 \sqrt{a D}}{1+a T / 2},
$$

where $D$ is given by Eq. (5). We use Eq. (20) to compute the second-order (HRD) prediction, using the same parameter values as above $[5,26]$. The result is shown in Fig. 1 as a dashed curve (HRD). It is seen that this second-order (HRD) approximation is not reliable, since its predicted speed is up to $31 \%$ less than that of the CSRW developed in Sec. II (and tested in Secs. III and IV) [3]. This shows very clearly the limitations of the HRD equation in Ref. [3], even for the very simple kernel considered (namely, that in which all moving individuals jump the same distance). We stress that we have chosen this kernel because we wanted to enquire the limitations of the approach in Ref. [3]. for a single, the simplest possible case. Clearly, in future work it would be interesting to extend our methods (CSRW, molecular-dynamics simulations and DSRW) to a variety of kernels appropriate for preindustrial agriculturalist societies.

In Ref. [3], we did not consider explicitly that some individuals or particles can remain at rest. Instead, all information about the dispersal kernel was averaged into a single parameter, namely, the diffusion coefficient (5). Therefore, it was not possible to analyze the effect of persistence on the predicted speed. In contrast, our new approach makes this possible. In Fig. 3, we compare the CSRW and simulation results for a range of values of the persistence consistent with the observed intergenerational mobility data of preindustrial farmers (see Sec. II B).

Originally the speed of the Neolithic transition in Europe was estimated as $0.8-1.2 \mathrm{~km} / \mathrm{yr}$, using a data set of only 53 archeological sites [41]. Over the years, a much larger data set has become available. Very recently, the $95 \%$-confidencelevel speed was estimated as $0.6-1.3 \mathrm{~km} / \mathrm{yr}$ using a data set of 735 sites [42]. The speeds predicted by our more accurate 
model (full curves in Figs. 1 and 3) are consistent with this observed range.

Figure 3 also shows that, over a wide range of persistence values, there is again good agreement between the CSRW model (full curves) and the molecular-dynamics simulations (triangles), for three different values of the net reproductive rate $R_{0}$. As in Fig. 1, we see from Fig. 3 that the HRD approximation (which was used in Ref. [3]) underestimates the exact results. This shows the interest of the work reported in this paper. Let us mention that its relevance is not restricted to the Neolithic transition, but it can also be of interest for other human range expansions, biological invasions, the spread of epidemics, etc.

The reason why the speed increases with increasing values of the persistence $p_{e}$ (Fig. 3) is that, for a given value of the diffusion coefficient (5), a higher value of the persistence implies that some individuals or families move larger distances per generation-so the reaction front moves faster, due to these long-range migration events. Similarly, the HRD speeds in Figs. 1 and 3 are lower because then the dispersion kernel is not considered except in an averaged way (as explained above), which ignores the fast component due to long-range dispersal events, thereby yielding a slower front.

\section{SEQUENTIAL REACTION-DIFFUSION EQUATION}

If we expand Eq. (10) up to second order in space and time, we will obtain the time-ordered analog to the HRD equation (3). We shall refer to the resulting equation as the SRD equation. If the SRD and the HRD results are similar, then we will conclude that the effect (ii) in Sec. I is negligible. If the SRD and the exact (CSRW) results are similar, then the effect (i) in Sec. I will be negligible. If the SRD result is substantially different from both the HRD and the exact results, then both effects (i) and (ii) will be important.

In order to derive the SRD equation, we approximate Eq. (10) by using Taylor expansions in space and time up to second order for isotropic kernels,

$$
p+T \frac{\partial p}{\partial t}+\frac{T^{2}}{2} \frac{\partial^{2} p}{\partial t^{2}}=R_{0} p+R_{0} D T\left(\frac{\partial^{2} p}{\partial x^{2}}+\frac{\partial^{2} p}{\partial y^{2}}\right),
$$

where $D$ is given by Eq. (5). Equation (21) is the SRD equation we wanted to derive. It is the time-ordered analog of the HRD equation (3). The speed of the SRD equation may be derived, as in Secs. II and IV, by assuming solutions with the form $p=p_{0} \exp [-\lambda(x-c t)]$ with $\lambda>0$. This yields

$$
\lambda=\frac{T c+\sqrt{(T c)^{2}-4\left(R_{0}-1\right)\left(R_{0} D T-\frac{T^{2} c^{2}}{2}\right)}}{2\left(R_{0} D T-\frac{T^{2} c^{2}}{2}\right)} .
$$

As in Secs. II and IV and Ref. [3], we assume that the minimum speed is the one of the front $[1,2]$. In this way we obtain the speed

$$
c_{\mathrm{SRD}}=\sqrt{\frac{2 R_{0} D}{T\left(1+\frac{1}{2\left(R_{0}-1\right)}\right)}} .
$$

In Figs. 1 and 3, we have also included this SRD speed (dashed-dotted curves). It is substantially different from both the HRD (dashed curves) and the exact, CSRW (full curves) speeds. We conclude that both effects (i) and (ii) are important for the purpose of analyzing the Neolithic transition. In fact, the SRD speed in Figs. 1 and 3 is closer to the exact speed (full curves) than the HRD equation used in Ref. [3] (dashed curves). This shows that the most relevant of both effects is not that of using second-order approximations [point (i) in Sec. I]. The most relevant effect is that of the time order or sequence of events [point (ii) in Sec. I]. Therefore, it is indeed important to take this effect into account, as done in the present paper.

\section{CONCLUDING REMARKS}

In this paper we have taken two effects into account: (i) not using second-order Taylor approximations and (ii) taking proper care of the order of events (i.e., the fact that children grow up with their parents before they become adults and can survive and migrate). We have presented three approaches to compute front speeds from the resulting integrodifference evolution equation: CSRWs (Sec. II), molecular-dynamics simulations, and DSRW (Sec. III). All of these approaches have lead us to front speeds consistent with each other (Figs. 1 and 3), as well as with the observed speed of the Neolithic transition in Europe. In contrast to previous work [3], which averaged the dispersal kernel into a single parameter (the diffusion coefficient), the models go beyond the second order [point (i) above] and thus allow us to analyze the speed of fronts as a function of the dispersion persistence of the population (Fig. 3).

We have also derived a time-ordered, second-order evolution equation, and called it the SRD equation. It has allowed us to conclude that the effect (ii) of the time order of events is more important than the effect (i) of the second-order Taylor approximations, but that both events should be considered in general.

The SRD equation agrees better with the exact, timeordered results than the HRD equation (Figs. 1 and 3). The SRD equation is therefore more realistic than the HRD equation (which was derived and applied in Ref. [3]). The reason is simply that the SRD equation is nothing but the timeordered equivalent to the HRD equation.

Differences between the new time-ordered model and the previously derived HRD equation [3] are as large as $70 \%$ (see Fig. 3). This percentage is important, and enough to be measurable, because it is 2 times as large as the uncertainty in the observed speed of the Neolithic front (see Sec. IV). Moreover, our results are also relevant to many biophysical phenomena other than the Neolithic transition, such as other human population range expansions, biological invasions, the spread of biological plagues, cultural fronts, tumor growth models, etc.

Let us finally stress that in this paper we have considered a simple kernel. In future work, it would be nice to extend the methods presented to a variety of dispersion kernels, which will in turn depend on the specific application considered. 


\section{ACKNOWLEDGMENTS}

Funded by the European Commission (Grant No. NEST28192-FEPRE), the MEC-FEDER (Grant No. FIS-200612296-C02-02), and the Generalitat de Catalunya (Grant No. SGR-2005-00087). One of the authors (N.I.) was supported by the MEC under the FPU program.

\section{APPENDIX A: AGE-STRUCTURED DERIVATION OF EQ. (1)}

Here we derive the nonsequential evolution equation (1) using an age-structured model. We generalize the framework in Ref. [5] to allow for age-dependant mortality and natality. For simplicity, here we consider a single value for the rest time $T$ between two successive migrations (i.e., $T$ $\simeq 1$ generation $\simeq 32$ years $[3,5]$ ) .

Let $\Sigma(a, x, y, t)$ stand for the number density (per unit area) of individuals aged $a$ that reach an area centered at point $(x, y)$ at time $t$. The total number of individuals $P(x, y, t)$ reaching the same location at $t$ is

$$
P(x, y, t)=\int_{0}^{\infty} d a \Sigma(a, x, y, t) .
$$

Let $\rho(a, x, y, t)$ stand for the number density (per unit area) of individuals aged $a$ at point $(x, y)$ at time $t$. The total number of individuals $p(x, y, t)$ at the same location at $t$ is

$$
p(x, y, t)=\int_{0}^{\infty} d a \rho(a, x, y, t) .
$$

Clearly, the generalizations of Eqs. (1) and (2) of Ref. [5] to include age structure are

$$
\begin{aligned}
& \Sigma(a, x, y, t)= \int_{0}^{t} d T \varphi(T) \int_{-\infty}^{+\infty} \int_{-\infty}^{+\infty} d \Delta_{x} d \Delta_{y} \Sigma\left(a-T, x+\Delta_{x}, y\right. \\
&\left.+\Delta_{y}, t-T\right) \phi\left(\Delta_{x}, \Delta_{y}\right)+\rho_{0}(a) \delta(x) \delta(y) \delta(t) \\
&+\delta(a) \int_{0}^{\infty} d a^{\prime} \lambda\left(a^{\prime}\right) \rho\left(a^{\prime}, x, y, t\right) \\
&-\mu_{0}(a) \rho(a, x, y, t) \\
& \rho(a, x, y, t)=\int_{0}^{t} d t^{\prime} \Sigma\left(a, x, y, t^{\prime}\right) \Psi\left(t-t^{\prime}\right)
\end{aligned}
$$

where, as in Ref. [5], $\varphi(T)$ is the probability that the particle or individual rests for a time between $T$ and $T+d T$ before performing the next jump, divided by $d T$. We have assumed that initially the density of individuals aged $a$ is $\rho_{0}(a)$ and vanishes outside the origin. $\lambda(a)$ and $\mu_{0}(a)$ are the natality and mortality rates. Higher-order mortality terms could be included, but are not necessary for our purposes (and there is not enough anthropological data to estimate them; anyway the front speed would be the same). We have also introduced [5]

$$
\Psi\left(t-t^{\prime}\right)=\int_{t-t^{\prime}}^{\infty} d T \varphi(T) .
$$

Integrating Eq. (A4) over age yields

$$
p(x, y, t)=\int_{0}^{t} d t^{\prime} P\left(x, y, t^{\prime}\right) \Psi\left(t-t^{\prime}\right) .
$$

Following Vlad $[43,44,4]$, we assume that the age structure reaches a stationary distribution $c_{s t}(a)$ which is also uniform in space, i.e.,

$$
\rho(a, x, y, t)=p(x, y, t) c_{s t}(a) .
$$

Integration of Eq. (A3) over age leads to

$$
\begin{aligned}
P(x, y, t)= & \int_{0}^{t} d T \varphi(T) \int_{-\infty}^{+\infty} \int_{-\infty}^{+\infty} d \Delta_{x} d \Delta_{y} P\left(x+\Delta_{x}, y\right. \\
& \left.+\Delta_{y}, t-T\right) \phi\left(\Delta_{x}, \Delta_{y}\right)+p_{0} \delta(x) \delta(y) \delta(t)+r_{L} p(x, y, t),
\end{aligned}
$$

where $p_{0}=\int_{0}^{\infty} d a \rho_{0}(a)$, and following Vlad $[43,44]$, we have introduced

$$
r_{L}=\int_{0}^{\infty} d a\left[\lambda(a)-\mu_{0}(a)\right] c_{s t}(a) .
$$

We now follow the same steps as in Ref. [5]. First, we Fourier-Laplace transform the previous equation,

$$
\hat{P}\left(k_{x}, k_{y}, s\right)=\hat{P}\left(k_{x}, k_{y}, s\right) \hat{\phi}\left(k_{x}, k_{y}\right) \hat{\varphi}(s)+p_{0}+\hat{N}\left(k_{x}, k_{y}, s\right),
$$

where

$$
\hat{N}\left(k_{x}, k_{y}, s\right)=r_{L} \hat{p}\left(k_{x}, k_{y}, s\right) .
$$

Second, we Fourier-Laplace transform Eq. (A6),

$$
\hat{p}\left(k_{x}, k_{y}, s\right)=\hat{P}\left(k_{x}, k_{y}, s\right) \frac{1-\hat{\varphi}(s)}{s} .
$$

Combining the two latter equations we get rid of the field $\hat{P}\left(k_{x}, k_{y}, s\right)$. Assuming a Dirac $\delta$ distribution for $\varphi(T)$, we have

$$
\frac{1}{\hat{\varphi}(s)}=\exp (s T)=1+\sum_{n=1}^{\infty} \frac{T^{n} s^{n}}{n !},
$$

and we obtain

$$
\begin{aligned}
\sum_{n=1}^{\infty} & \frac{T^{n} s^{n-1}}{n !}\left[s \hat{p}\left(k_{x}, k_{y}, s\right)-p_{0}\right] \\
\quad & =\left[\hat{\phi}\left(k_{x}, k_{y}\right)-1\right] \hat{p}\left(k_{x}, k_{y}, s\right)+\sum_{n=1}^{\infty} \frac{T^{n} s^{n-1}}{n !} \hat{N}\left(k_{x}, k_{y}, s\right) .
\end{aligned}
$$

Antitransforming this equation yields 


$$
\begin{aligned}
\sum_{n=1}^{\infty} \frac{T^{n}}{n !} \frac{\partial^{n} p}{\partial t^{n}}= & \int_{-\infty}^{+\infty} \int_{-\infty}^{+\infty} p\left(x+\Delta_{x}, y+\Delta_{y}, t\right) \phi\left(\Delta_{x}, \Delta_{y}\right) d \Delta_{x} d \Delta_{y} \\
& -p(x, y, t)+\sum_{n=1}^{\infty} \frac{T^{n}}{n !} \frac{\partial^{n-1} N(x, y, t)}{\partial t^{n-1}} .
\end{aligned}
$$

From Eq. (A11),

$$
N(x, y, t)=r_{L} p(x, y, t) .
$$

Using this and introducing the so-called exponential growth function $F[p(x, y, t)]=r_{L} p(x, y, t)$, we obtain

$$
\begin{aligned}
p(x, y, t+T)-p(x, y, t)= & \int_{-\infty}^{+\infty} \int_{-\infty}^{+\infty} p\left(x+\Delta_{x}, y\right. \\
& \left.+\Delta_{y}, t\right) \phi\left(\Delta_{x}, \Delta_{y}\right) d \Delta_{x} d \Delta_{y}-p(x, y, t) \\
& +\sum_{n=1}^{\infty} \frac{T^{n}}{n !} \frac{\partial^{n-1} F[p(x, y, t)]}{\partial t^{n-1}},
\end{aligned}
$$

so that using Eq. (4), we obtain

$$
\begin{aligned}
p(x, y, t+T)-p(x, y, t)= & \int_{-\infty}^{+\infty} \int_{-\infty}^{+\infty} p\left(x+\Delta_{x}, y\right. \\
& \left.+\Delta_{y}, t\right) \phi\left(\Delta_{x}, \Delta_{y}\right) d \Delta_{x} d \Delta_{y}-p(x, y, t) \\
& +R[p(x, y, t)] .
\end{aligned}
$$

This completes the age-structured derivation of Eq. (1).

It is possible to add a quadratic mortality term, e.g., $-\mu_{1} p(x, y, t) \rho(a, x, y, t)$ [44], to Eq. (A3), which yields Eq. (1) with a logistic (instead of an exponential) growth function $F[p(x, y, t)]$. However, this is not necessary for the purposes of the present paper (and the speed of front solutions would be the same).

\section{APPENDIX B: AGE-STRUCTURED DERIVATION OF EQ. (6)}

Here we derive the sequential (or time-ordered) evolution Eq. (6) using an age-structured model. For this purpose, we modify Appendix A to take into account that for human populations, parents do not leave their sons and daughters until the latter become adults and can survive (and migrate). Then Eq. (A3) is replaced by

$$
\begin{aligned}
\Sigma(a, x, y, t)= & \int_{0}^{t} d T \varphi(T) \int_{-\infty}^{+\infty} \int_{-\infty}^{+\infty} d \Delta_{x} d \Delta_{y} \Sigma\left(a-T, x+\Delta_{x}, y\right. \\
& \left.+\Delta_{y}, t-T\right) \phi\left(\Delta_{x}, \Delta_{y}\right)+\rho_{0}(a) \delta(x) \delta(y) \delta(t) \\
& +\delta(a) \int_{0}^{\infty} d a^{\prime} \lambda\left(a^{\prime}\right) \int_{-\infty}^{+\infty} \int_{-\infty}^{+\infty} d \Delta_{x} d \Delta_{y} \\
& \times \rho\left(a^{\prime}, x+\Delta_{x}, y+\Delta_{y}, t\right) \phi\left(\Delta_{x}, \Delta_{y}\right) \\
& -\mu_{0}(a) \int_{-\infty}^{+\infty} \int_{-\infty}^{+\infty} d \Delta_{x} d \Delta_{y} \\
& \times \rho\left(a, x+\Delta_{x}, y+\Delta_{y}, t\right) \phi\left(\Delta_{x}, \Delta_{y}\right),
\end{aligned}
$$

so that net reproduction (last two terms) takes place at the arrival location. It is thus clear that the difference between Eqs. (B1) and (A3) is precisely the same as that between Eqs. (6) and (1).

It is easy to repeat all steps in Appendix A. The only difference is that Eq. (A11) is replaced by

$$
\hat{N}\left(k_{x}, k_{y}, s\right)=r_{L} \hat{p}\left(k_{x}, k_{y}, s\right) \hat{\phi}\left(k_{x}, k_{y}\right),
$$

and Eq. (A14) remains valid. However, instead of Eq. (A15) we obtain

$$
\begin{aligned}
\sum_{n=1}^{\infty} \frac{T^{n}}{n !} & \frac{\partial^{n} p(x, y, t)}{\partial t^{n}} \\
= & \int_{-\infty}^{+\infty} \int_{-\infty}^{+\infty} p\left(x+\Delta_{x}, y+\Delta_{y}, t\right) \phi\left(\Delta_{x}, \Delta_{y}\right) d \Delta_{x} d \Delta_{y} \\
& -p(x, y, t)+r_{L} \int_{-\infty}^{+\infty} \int_{-\infty}^{+\infty} d \Delta_{x} d \Delta_{y} \phi\left(\Delta_{x}, \Delta_{y}\right) \\
& \times\left(T p\left(x+\Delta_{x}, y+\Delta_{y}, t\right)\right. \\
& \left.+\frac{T^{2}}{2 !} \frac{\partial p\left(x+\Delta_{x}, y+\Delta_{y}, t\right)}{\partial t}+\cdots\right) .
\end{aligned}
$$

As in Appendix A, we introduce the exponential growth function $F[p(x, y, t)]=r_{L} p(x, y, t)$. From the previous equation we obtain

$$
\begin{aligned}
p(x, y, t & +T)-p(x, y, t) \\
= & \int_{-\infty}^{+\infty} \int_{-\infty}^{+\infty} p\left(x+\Delta_{x}, y+\Delta_{y}, t\right) \phi\left(\Delta_{x}, \Delta_{y}\right) \\
& \times d \Delta_{x} d \Delta_{y}-p(x, y, t) \\
& +T F\left(\int_{-\infty}^{+\infty} \int_{-\infty}^{+\infty} d \Delta_{x} d \Delta_{y} \phi\left(\Delta_{x}, \Delta_{y}\right) p\left(x+\Delta_{x}, y+\Delta_{y}, t\right)\right) \\
& +\frac{T^{2}}{2 !} \frac{\partial F}{\partial t}\left(\int_{-\infty}^{+\infty} \int_{-\infty}^{+\infty} d \Delta_{x} d \Delta_{y} \phi\left(\Delta_{x}, \Delta_{y}\right)\right. \\
& \left.\times p\left(x+\Delta_{x}, y+\Delta_{y}, t\right)\right)+\cdots
\end{aligned}
$$

and, using Eq. (4) as in Appendix A, we obtain now

$$
\begin{aligned}
p(x, y, t+T)-p(x, y, t) & \\
= & \int_{-\infty}^{+\infty} \int_{-\infty}^{+\infty} p\left(x+\Delta_{x}, y+\Delta_{y}, t\right) \phi\left(\Delta_{x}, \Delta_{y}\right) \\
& \times d \Delta_{x} d \Delta_{y}-p(x, y, t) \\
& +R\left(\int_{-\infty}^{+\infty} \int_{-\infty}^{+\infty} p\left(x+\Delta_{x}, y+\Delta_{y}, t\right) \phi\left(\Delta_{x}, \Delta_{y}\right) d \Delta_{x} d \Delta_{y}\right) .
\end{aligned}
$$

This completes the age-structured derivation of Eq. (6).

As in Appendix A, it is possible to add a quadratic mortality term, e.g., $\quad-\mu_{1} \int_{-\infty}^{+\infty} \int_{-\infty}^{+\infty} d \Delta_{x} d \Delta_{y} p\left(x+\Delta_{x}, y\right.$ 
$\left.+\Delta_{y}, t\right) \phi\left(\Delta_{x}, \Delta_{y}\right) \int_{-\infty}^{+\infty} \int_{-\infty}^{+\infty} d \Delta_{x} d \Delta_{y} \rho\left(a, x+\Delta_{x}, y+\Delta_{y}, t\right) \phi\left(\Delta_{x}, \Delta_{y}\right)$, to Eq. (B1), which yields Eq. (6) with a logistic (instead of an exponential) growth function $F[p(x, y, t)]$. But again, this is not necessary for the purposes of the present paper (and the speed of front solutions would be the same).

\section{APPENDIX C: AGE-STRUCTURED DERIVATION OF EQ. (10)}

In Appendixes $\mathrm{A}$ and $\mathrm{B}$, we have used mortality and natality instantaneous rates, $\mu_{0}(a)$ and $\lambda(a)$, respectively. Here we show that using mortality and natality rates per generation, $\widetilde{\mu}_{0}(a)$ and $\tilde{\lambda}(a)$, respectively, it is possible to derive Eq. (10). Using such rates, we may write down the following simple sequential equation

$$
\begin{aligned}
& \rho(a+T, x, y, t+T)-\rho(a, x, y, t) \\
&=+\int_{-\infty}^{+\infty} \int_{-\infty}^{+\infty} d \Delta_{x} d \Delta_{y} \rho\left(a, x+\Delta_{x}, y+\Delta_{y}, t\right) \phi\left(\Delta_{x}, \Delta_{y}\right) \\
&-\rho(a, x, y, t)-\tilde{\mu}_{0}(a) \int_{-\infty}^{+\infty} \int_{-\infty}^{+\infty} d \Delta_{x} d \Delta_{y} \rho \\
& \times\left(a, x+\Delta_{x}, y+\Delta_{y}, t\right) \phi\left(\Delta_{x}, \Delta_{y}\right),
\end{aligned}
$$

so that mortality (last term) takes place at the arrival location [as in Appendix B and Eq. (6)].

Integrating this equation over age ( $a=0$ to $a=\infty)$ and defining $a^{\prime} \equiv a+T$, we obtain

$$
\begin{aligned}
& p(x, y, t+T)-\int_{0}^{T} d a^{\prime} \rho\left(a^{\prime}, x, y, t+T\right)-p(x, y, t) \\
& =\int_{-\infty}^{+\infty} \int_{-\infty}^{+\infty} d \Delta_{x} d \Delta_{y} p\left(x+\Delta_{x}, y+\Delta_{y}, t\right) \phi\left(\Delta_{x}, \Delta_{y}\right) \\
& \quad-p(x, y, t)-\int_{0}^{\infty} d a \tilde{\mu}_{0}(a) \int_{-\infty}^{+\infty} \int_{-\infty}^{+\infty} d \Delta_{x} d \Delta_{y} \\
& \quad \times \rho\left(a, x+\Delta_{x}, y+\Delta_{y}, t\right) \phi\left(\Delta_{x}, \Delta_{y}\right) .
\end{aligned}
$$

The second term corresponds obviously to individuals with ages between 0 and $T$, i.e., born between $t$ and $t+T$, namely $\int_{0}^{\infty} d a \tilde{\lambda}(a) \int_{-\infty}^{+\infty} \int_{-\infty}^{+\infty} d \Delta_{x} d \Delta_{y} \rho\left(a, x+\Delta_{x}, y+\Delta_{y}, t\right) \phi\left(\Delta_{x}, \Delta_{y}\right) \quad[$ because $T$ is one generation and $\tilde{\lambda}(a)$ is the birth rate per generation]. Again as in Appendix B and Eq. (6), reproduction takes place at the arrival location, so that parents do not migrate away from their children. It means that children grow up with their parents, before becoming adults and able to survive and migrate themselves (in this sense, this is a sequential or time-ordered model-as is Appendix B). Using also Eq. (A7) leads us finally to Eq. (10), namely,

$$
\begin{aligned}
& p(x, y, t+T) \\
& \quad=R_{0} \int_{-\infty}^{+\infty} \int_{-\infty}^{+\infty} d \Delta_{x} d \Delta_{y} p\left(x+\Delta_{x}, y+\Delta_{y}, t\right) \phi\left(\Delta_{x}, \Delta_{y}\right),
\end{aligned}
$$

where we have defined $R_{0}$ as

$$
R_{0}-1 \equiv \int_{0}^{\infty} d a\left[\tilde{\lambda}(a)-\tilde{\mu}_{0}(a)\right] c_{s t}(a) .
$$

We note that the condition $R_{0}>1$, which Weinberger [20] showed that is necessary for the population not to extinguish and front solutions to Eq. (10) to exist, corresponds to the effect of natality being stronger than that of mortality, which makes biological sense.

As in Appendixes $\mathrm{A}$ and $\mathrm{B}$, it is possible to add a quadratic term, e.g., $\quad-\widetilde{\mu}_{1} \int_{-\infty}^{+\infty} \int_{-\infty}^{+\infty} d \Delta_{x} d \Delta_{y} p\left(x+\Delta_{x}, y\right.$ $\left.+\Delta_{y}, t\right) \phi\left(\Delta_{x}, \Delta_{y}\right) \int_{-\infty}^{+\infty} \int_{-\infty}^{+\infty} d \Delta_{x} d \Delta_{y} \rho\left(a, x+\Delta_{x}, y+\Delta_{y}, t\right) \phi\left(\Delta_{x}, \Delta_{y}\right)$, to Eq. (C1), which yields a logistic discrete-time reproduction function $R[p(x, y, t)]$, i.e., an additional term $-\widetilde{\mu}_{1}\left[\int_{-\infty}^{+\infty} \int_{-\infty}^{+\infty} d \Delta_{x} d \Delta_{y} p\left(x+\Delta_{x}, y+\Delta_{y}, t\right) \phi\left(\Delta_{x}, \Delta_{y}\right)\right]^{2}$ to Eq. (C4). However, this is not necessary for the purposes of the present paper, the speed of fronts would be the same and, more importantly, a logistic discrete-time reproduction function $R[p(x, y, t)]$ is known from nonspatial models to yield negative population densities [40], which makes no physical sense [we have found via numerical simulations (similar to those in Sec. III A) that the same happens for spatial models]. This is the reason why we use Eq. (10) and vanishing net reproduction above saturation density [see the second line in Eq. (9) and point (i) in Sec. III A].
[1] J. Fort and V. Méndez, Rep. Prog. Phys. 65, 895 (2002).

[2] W. van Saarloos, Phys. Rep. 386, 29 (2003).

[3] J. Fort and V. Méndez, Phys. Rev. Lett. 82, 867 (1999). In this reference, the mean-squared displacement was estimated as $\left\langle\Delta^{2}\right\rangle=1544 \mathrm{~km}^{2}$ (using mobility data from Refs. [39,31]).

[4] M. O. Vlad and J. Ross, Phys. Rev. E 66, 061908 (2002).

[5] J. Fort, D. Jana, and J. Humet, Phys. Rev. E 70, 031913 (2004). For the estimation $T=32 \mathrm{yr}$ of the generation time, see note $[24]$ in this reference.

[6] K. Davison, P. Dolukhanov, G. R. Sarson, and A. Shukurov, J. Archaeol. Sci. 33, 641 (2006).

[7] S. Harris, Phys. Rev. E 68, 031912 (2003).
[8] M. H. Cohen, in Nonlinearity with Disorder, edited by F. Adbullaev, A. R. Bishop, and S. Pnevmatikos (Springer, Berlin, 1992), pp. 161-170.

[9] M. O. Vlad, L. L. Cavalli-Sforza, and J. Ross, Proc. Natl. Acad. Sci. U.S.A. 101, 10249 (2004).

[10] J. Fort and V. Méndez, Phys. Rev. Lett. 89, 178101 (2002).

[11] J. Fort, J. Pérez, E. Ubeda, and F. J. García, Phys. Rev. E 73, 021907 (2006).

[12] N. Shigesada and K. Kawasaki, Biological Invasions. Theory and Practice (Oxford University Press, Oxford, 1997).

[13] J. Merikoski, J. Maunuksela, M. Myllys, J. Timonen, and M. J. Alava, Phys. Rev. Lett. 90, 024501 (2003). 
[14] J. Fort, T. Pujol, and A. S. Cukrowski, J. Phys. A 33, 6953 (2000).

[15] J. Fort, D. Campos, J. R. González, and J. Velayos, J. Phys. A 37, 7185 (2004).

[16] D. Campos, J. E. Llebot, and J. Fort, J. Phys. A 37, 6609 (2004)

[17] A. Brú, J. M. Pastor, I. Fernaud, I. Brú, S. Melle, and C. Berenguer, Phys. Rev. Lett. 81, 4008 (1998).

[18] A. Brú, S. Albertos, J. A. López García-Asenjo, and I. Brú, Phys. Rev. Lett. 92, 238101 (2004).

[19] S. Fedotov and A. Iomin, Phys. Rev. Lett. 98, 118101 (2007).

[20] H. F. Weinberger, in Nonlinear Partial Differential Equations and Applications, edited by J. Chadam (Springer, Berlin, 1978).

[21] J. S. Clark, Am. Nat. 152, 204 (1998).

[22] J. Fort and V. Méndez, Phys. Rev. E 60, 5894 (1999).

[23] V. Méndez, T. Pujol, and J. Fort, Phys. Rev. E 65, 041109 (2002).

[24] V. Méndez, D. Campos, and J. Fort, Europhys. Lett. 66, 902 (2004).

[25] Observed saturation densities for agriculturalist populations are in the range $3-300$ people $/ \mathrm{km}^{2}$, depending on the environment and technology [39]. However, the front speed is independent of this numerical value.

[26] The connection between the low-density growth parameters $a$ in the logistic equation (8) and $R_{0}$ in Eq. (9) can be obtained most easily as follows. In the absence of dispersal, both Eqs. (1) and (6) become $p(x, y, t+T)-p(x, y, t)=R[p(x, y, t)]$ which, combined with Eq. (4), implies that $\partial p / \partial t=F$. Thus, for low values of $p(x, y, t)$, the logistic form (8) yields $p(x, y, t+T)$ $=p(x, y, t) \exp (a T)$, whereas Eq. (9) yields $p(x, y, t+T)$ $=R_{0} p(x, y, T)$. Comparing both expressions, we see that $a$ $=(1 / T) \ln R_{0}$.

[27] This is easily seen from either of both analytical approaches in this paper (CSRWs and DSRWs).

[28] C. A. S. Hall, Ecol. Modell. 43, 5 (1988).

[29] A simple way to see this is to note that the mean values are $\int_{-\infty}^{\infty} d \Delta_{x} \int_{-\infty}^{\infty} d \Delta_{y} \Delta \delta^{(2)}(\Delta-r)=\int_{0}^{2 \pi} d \theta \int_{0}^{\infty} d \Delta \Delta^{2} \delta^{(2)}(\Delta-r)=r \quad$ and $\int_{0}^{\infty} d \Delta \Delta \delta^{(1)}(\Delta-r)=r$, whereas the corresponding normalization conditions are $\int_{0}^{2 \pi} d \theta \int_{0}^{\infty} d \Delta \Delta \delta^{(2)}(\Delta-r)=1$ and $\int_{0}^{\infty} d \Delta \delta^{(1)}(\Delta-r)$ $=1$.

[30] J. P. Birdsell, Cold Spring Harb. Symp. Quant. Biol. 22, 47 (1957)

[31] J. Stauder, The Majangir. Ecology and Society of a Southwest Ethiopian People (Cambridge University Press, Cambridge, 1971).

[32] We have computed the value of $r$ such that the mean-squared displacement yields the observed value (namely $1544 \mathrm{~km}^{2}$ [3]), i.e., $\left(1-p_{e}\right) r^{2}=\left\langle\Delta^{2}\right\rangle=1544 \mathrm{~km}^{2}$.

[33] V. Méndez, J. Fort, and J. Farjas, Phys. Rev. E 60, 5231 (1999).

[34] V. Méndez and J. Fort, Phys. Rev. E 64, 011105 (2001).

[35] V. Méndez, J. Fort, H. G. Rotstein, and S. Fedotov, Phys. Rev. E 68, 041105 (2003).

[36] V. Ortega-Cejas, J. Fort, V. Méndez, and D. Campos, Phys. Rev. E 69, 031909 (2004).

[37] One could estimate the speed in other directions. However, such substantially more tedious work is unnecessary because of the satisfactory agreement between the CSRW (full line in Fig. 1) and the mean of the simulations (triangles).

[38] Note that the generation time in Ref. [3] was estimated as $T$ $=25 \mathrm{yr}$, directly from Ref. [39]. But very recently it has been noted that this value, as used in demography, is the mean age difference between parents and their oldest son or daughter (not the mean among all of them). Thus, a more precise estimation is $T=32 \mathrm{yr}$ [5]. This is why the HRD curve in Fig. 1 in the present paper for the value $a=0.03 \mathrm{yr}^{-1}$, considered in [3] [i.e., for $R_{0}=\exp (a T)=2.61$ [26]], yields a slower front than that in Ref. [3] (0.81 km/yr versus $0.99 \mathrm{~km} / \mathrm{yr})$.

[39] A. J. Ammerman and L. L. Cavalli-Sforza, The Neolithic Transition and the Genetics of Population in Europe (Princeton University Press, Princeton, NJ, 1984).

[40] J. D. Murray, Mathematical Biology, 3rd ed. (Springer-Verlag, Berlin, 2002), Sec. 2.1.

[41] A. J. Ammerman and L. L. Cavalli-Sforza, Man 6, 674 (1971).

[42] R. Pinhasi, J. Fort, and A. J. Ammerman, PLoS Biol. 3, e410 (2005).

[43] M. O. Vlad and V. T. Popa, Math. Biosci. 76, 161 (1985).

[44] M. O. Vlad, J. Theor. Biol. 126, 239 (1987). 